\title{
IDENTIFYING MULTI-ATTRIBUTE HEDONIC MODELS
}

\author{
VICTOR CHERNOZHUKOV, ALFRED GALICHON AND MARC HENRY
}

\begin{abstract}
This paper derives conditions under which preferences and technology are nonparametrically identified in hedonic equilibrium models, where products are differentiated along more than one dimension and agents are characterized by several dimensions of unobserved heterogeneity. With products differentiated along a quality index and agents characterized by scalar unobserved heterogeneity, single crossing conditions on preferences and technology provide identifying restrictions. We develop similar shape restrictions in the multi-attribute case and we provide identification results from the observation of a single market. We thereby extend identification results in Matzkin (2003) and Heckman, Matzkin, and Nesheim (2010) to accommodate multiple dimensions of unobserved heterogeneity. With an exclusion restriction or multiple market data, we further show identification of marginal effects, when the distribution of unobserved tastes for quality is unspecified.
\end{abstract}

Keywords: Hedonic equilibrium, nonparametric identification, multidimensional unobserved heterogeneity, optimal transport.

JEL subject classification: C14, C61, C78

Date: The present version is of May 29, 2014. The authors thank Guillaume Carlier and conference participants in Oberwolfach for stimulating discussions. Galichon's research has received funding from the European Research Council under the European Union's Seventh Framework Programme (FP7/20072013), ERC grant agreement no 313699, and from FiME, Laboratoire de Finance des Marchés de l'Energie. Henry's research has received funding from SSHRC Grants 410-2010-242 and 435-2013-0292 and NSERC Grant 356491-2013. 


\section{INTRODUCTION}

Recent years have seen renewed interest in hedonic models, particularly their identification and estimation. Hedonic models were initially introduced to analyze price responses to quality parameters of differentiated goods. Among these: (1) Given the fact that the amenities offered by cars constantly evolve over time, how can one construct a price index measuring the evolution of the car prices? (2) How can one explain price differentiation in wine, art, luxury goods, professional sports wages? (3) What does the correlation between the wage differentials and the level of risk associated to a given job reveal about individuals' valuation for their own life? (4) How can one analyze individual preferences for environmental features?

These questions gave rise to a vast literature, which aims at modeling implicit markets for quality differentiated products. There are two layers to this literature. The first layer is the literature on "hedonic regressions," which aims at estimating consumer willingness to pay for quality, while correcting for the standard endogeneity issue that consumers with greater taste for quality will consume more of it. The second layer, which concerns us here, has broader scope: the literature on "hedonic equilibrium models" incorporates a supply side with differentiated productivity over various quality parameters and studies the resulting equilibrium. This approach dates back at least as far as Tinbergen (1956); and Rosen (1974) provides a famous two-step procedure to estimate general hedonic models and thereby analyze general equilibrium effects of changes in buyer-seller compositions, preferences and technology on qualities traded at equilibrium and their price. Following the influential criticism of Rosen's strategy in Brown and Rosen (1982) and the inadmissibility of supply side observable characteristics as instruments in structural demand estimation as discussed in Epple (1987) and Bartik (1987), it was generally believed that identification in hedonic equilibrium models required data from multiple markets, as in Epple (1987), Khan 
and Lang (1988) and, more recently, Bajari and Benkard (2005) and Bishop and Timmins (2011).

Ekeland, Heckman, and Nesheim (2004) show, however, that hedonic equilibrium models are in fact identified from single market data, under separability assumptions, as in Ekeland, Heckman, and Nesheim (2004), or shape restrictions, as in Heckman, Matzkin, and Nesheim (2010). The common underlying framework is that of a perfectly competitive market with heterogeneous buyers and sellers and traded product quality bundles and prices that arise endogeneously in equilibrium. Preferences are quasi-linear in price and under mild semicontinuity assumptions, Ekeland (2010) and Chiappori, McCann, and Nesheim (2010) show that equilibria exist, in the form of a joint distribution of product and consumer types (who consumes what), a joint distribution of product and producer types (who produces what) and a price schedule such that markets clear for each endogenously traded product type. Equilibrium existence results are valid in hedonic markets for multi-attribute products, but existing single market identification strategies restrict attention to a single quality dimension and scalar unobserved heterogeneity in consumer preferences and production technology. Ekeland, Heckman, and Nesheim (2004) require marginal utility (resp. marginal product) to be additively separable in unobserved consumer (resp. producer) characteristic. Heckman, Matzkin, and Nesheim (2010) show that demand is nonparametrically identified under a single crossing condition and that various additional shape restrictions allow identification of preferences without additive separability.

The objective of these papers and ours is to recover structural preference and technology parameters from the observation of who trades what and at what price. In the identification exercise, price is assumed known, as are the distributions characterizing who produces and consumes which good. Since price is observed and the environment is perfectly competitive, identification of preferences and identification of technology can be treated independently and symmetrically. Take the consumer problem, for instance. Under a single crossing condition on the utility function (also known as 
Spence-Mirlees in the mechanism design literature), the first order condition of the consumer problem yields an increasing demand function, i.e., quality demanded by the consumer as an increasing function of her unobserved type, interpreted as unobserved taste for quality. Assortative matching guarantees uniqueness of demand, as the unique increasing function that maps the distribution of unobserved taste for quality, which is specified a priori, and the distribution of qualities, which is observed. Hence demand is identified as a quantile function, as in Matzkin (2003). Identification, therefore, is driven by a shape restriction on the utility function. We show that similar shape restrictions on the utility function also yield identification conditions in the case of non scalar characteristics and unobserved heterogeneity. In the special case, where marginal utility is additively separable in the unobservable taste vector, concavity yields nonparametric identification of the utility function, according to the celebrated Brenier Theorem of optimal transport theory (Theorem 3.8 of Villani (2003)). More generally, a generalization of single crossing known as the Twist Condition in optimal transport theory and a generalized convexity shape restriction yield identification of the utility function in hedonic equilibrium models with multiple quality dimensions. The distribution of unobserved heterogeneity is fully specified a priori and cannot be identified from single market data. However, we show that additional restrictions or data from multiple markets provide identifying power when the distribution of unobserved tastes is unknown. We derive specific identification results when marginal utilities are additively separable in consumer and good characteristics, on the one hand, and when a variable (market index) shifts quality distributions and prices, but is excluded from preferences, on the other hand.

Related work. Beyond Ekeland, Heckman, and Nesheim (2004), Heckman, Matzkin, and Nesheim (2010) and other contributions cited so far, this paper is closely related to the growing literature on identification and estimation of nonlinear econometric 
models with multivariate unobserved heterogeneity on the one hand, and to the empirical literature on matching models where agents match along multiple dimensions on the other hand. The quantile identification strategy of Matzkin (2003) was recently extended to non scalar unobserved heterogeneity using the Rosenblatt (1952)-Knothe (1957) sequential multivariate quantile transform for nonlinear simultaneous equations models in Matzkin (2013) and bivariate hedonic models in Nesheim (2013). Chiappori, McCann, and Nesheim (2010) derive a matching formulation of hedonic models and thereby highlight the close relation between empirical strategies in matching markets and in hedonic markets. Galichon and Salanié (2012) extend the work of Choo and Siow (2006) and identify preferences in marriage markets, where agents match on discrete characteristics, as the unique solution of a programming problem, as in the present paper. The strategy is also applied in Chiong, Galichon, and Shum (2013) for identification of dynamic discrete choice problems.

Organization of the paper. The remainder of the paper is organized as follows. Section 2 sets the hedonic equilibrium framework. Section 3 gives an account of the main results on nonparametric identification of preferences in single attribute hedonic models, mostly drawn from Ekeland, Heckman, and Nesheim (2004) and Heckman, Matzkin, and Nesheim (2010). Section 4 is the main section of the paper and shows how these results and the shape restrictions that drive them can be extended to the case of multiple attribute hedonic equilibrium markets. Section 5 shows how multiple market data can be brought to bear on identifying the distribution of unobserved heterogeneity. The last section concludes and discusses future research directions. Proofs of the main results are relegated to the appendix, as are background results on optimal transport theory and hedonic equilibrium theory. 


\section{Hedonic Equilibrium AND the identificAtion PROBlem}

We consider a competitive environment, where consumers and producers trade a good or contract, fully characterized by its type or quality $z$. The set of feasible qualities $Z \subseteq \mathbb{R}^{d_{z}}$ is given, but the qualities actually traded arise endogenously in the hedonic market equilibrium, as does their price schedule $p(z)$. Producers are characterized by their type $\tilde{y} \in \tilde{Y} \subseteq \mathbb{R}^{d_{\tilde{y}}}$ and consumers by their type $\tilde{x} \in \tilde{X} \subseteq \mathbb{R}^{d_{\tilde{x}}}$. Type distributions $P_{\tilde{x}}$ on $\tilde{X}$ and $P_{\tilde{y}}$ on $\tilde{Y}$ are given exogenously, so that entry and exit are not modelled, except in the possibility of non participation, which is modelled by adding isolated points to the sets of types and renormalizing distributions accordingly (see Section 1.1 of Chiappori, McCann, and Nesheim (2010) for details). Consumers and producers are price takers and maximize quasi-linear utility $U(\tilde{x}, z)-p(z)$ and profit $p(z)-C(\tilde{y}, z)$ respectively. Utility $U(\tilde{x}, z)$ (respectively cost $C(\tilde{y}, z)$ ) is upper (respectively lower) semicontinuous and bounded and normalized to zero in case of nonparticipation. In addition, the set of qualities $Z(\tilde{x}, \tilde{y})$ that maximize the surplus $U(\tilde{x}, z)-C(\tilde{y}, z)$ for each pair of types $(\tilde{x}, \tilde{y})$ is assumed to have a measurable selection. Then, Ekeland (2010) and Chiappori, McCann, and Nesheim (2010) show that an equilibrium exists in this market, in the form of a price function $p$ on $Z$, a joint distribution $P_{\tilde{x}, z}$ on $\tilde{X} \times Z$ and $P_{\tilde{y}, z}$ on $\tilde{Y} \times Z$ such that their marginal on $Z$ coincide, so that market clears for each traded quality $z \in Z$. Uniqueness is not guaranteed, in particular prices are not uniquely defined for non traded quantities in equilibrium. Purity is not guaranteed either: an equilibrium specifies a conditional distribution $P_{z \mid \tilde{x}}$ (respectively $P_{z \mid \tilde{y}}$ ) of qualities consumed by type $\tilde{x}$ consumers (respectively produced by type $\tilde{y}$ producers). The quality traded by a given producer-consumer pair $(\tilde{x}, \tilde{y})$ is not uniquely determined at equilibrium.

Ekeland (2010) and Chiappori, McCann, and Nesheim (2010) further show that a pure equilibrium exists and is unique, under the additional assumption that type distributions $P_{\tilde{x}}$ and $P_{\tilde{y}}$ are absolutely continuous and gradients of utility and cost, 
$\nabla_{\tilde{x}} U(\tilde{x}, z)$ and $\nabla_{\tilde{y}} C(\tilde{y}, z)$ exist and are injective as functions of quality $z$. The latter condition, also known as the Twist condition in the optimal transport literature, ensures that a consumer of a given type $\tilde{x}$ (respectively producer of a given type $\tilde{y}$ ) will always consume (respectively produce) the same quality $z$ at equilibrium. This will turn out to be a crucial aspect of the identification strategy as discussed next.

The identification problem consists in the recovery of structural features of preferences and technology from observation of traded quantities and their prices in a single market, in a first stage, and with data from multiple markets, in a second stage. Given observability of prices and the fact that producer type $\tilde{y}$ (respectively consumer type $\tilde{x})$ does not enter into the utility function $U(\tilde{x}, z)$ (respectively cost function $C(\tilde{y}, z)$ ) directly, we may consider the consumer and producer problems separately and symmetrically. We focus on the consumer problem and on identification of utility function $U(\tilde{x}, z)$. Under assumptions ensuring purity and uniqueness of equilibrium, the model predicts a deterministic choice of quality $z$ for a given consumer type $\tilde{x}$. Hence, to preclude outright rejection of the model with any data set, where identical observable types consume different goods, we assume, as is customary, that consumer types $\tilde{x}$ are only partially observable to the analyst. We write $\tilde{x}=(x, \varepsilon)$, where $x \in \mathbb{R}^{d_{x}}$ is the observable part of the type vector, and $\varepsilon \in \mathbb{R}^{d_{\varepsilon}}$ is the unobservable part. The observable and unobservable types will be assumed independant and we shall make a separability assumption that will allow us to specify constraints on the interaction between consumer unobservable type $\varepsilon$ and good quality $z$ in order to identify interactions between observable type $x$ and good quality $z$.

Assumption 1 (Unobservable heterogeneity). Consumer type $\tilde{x}$ is composed of observable type $x$ with distribution $P_{x}$ on $\mathbb{R}^{d_{x}}$ and unobservable type $\varepsilon$ with a priori specified distribution $P_{\varepsilon}$ on $\mathbb{R}^{d_{\varepsilon}}$. Observable and unobservable types are independent and the utility of consumers can be decomposed as $U(\tilde{x}, z)=\bar{U}(x, z)+\zeta(x, \varepsilon, z)$. 
Assumption 1 includes independance of observable and unobservable types, which, although a strong requirement, is necessary in identification strategies that extend the quantile identification of Matzkin (2003). Specification of $P_{\varepsilon}$ is a necessary normalization, which also stems from quantile identification. Note that the separability assumption added here is vacuous until restrictions on the function $\zeta$ are specified.

We shall work throughout under the following condition on the distribution of traded qualities.

Assumption 2 (Distribution of traded qualities). For all $x, P_{z \mid x}$ is absolutely continuous with respect to Lebesgue measure.

This assumption is not a primitive assumption on the distributions of producers and consumers and as such. It can be relaxed somewhat to a weaker condition, namely that $P_{z \mid x}$ does not give mass to sets of Hausdorff dimension at most $d_{z}-1$ in $\mathbb{R}^{d_{z}}$, but it cannot be dispensed with altogether, as we see in the proof of Theorem 2. Primitive conditions are given in Pass (2011) that ensure that the support of the distribution of endogenously traded qualities has Hausdorff dimension $d_{z}$.

The object of inference is the deterministic component of utility $\bar{U}(x, z)$. We shall denote

$$
V(x, z)=p(z)-\bar{U}(x, z)
$$

and focus on identification of the function $V$, since under observability of price, it is equivalent to identification of the deterministic part of utility. We shall identify $\bar{U}(x, z)$ up to a constant and use the following terminology.

Definition 1 (Nonparametric identification). The function $\bar{U}(x, z)$ will be called nonparametrically identified if it exists and $\nabla_{z} \bar{U}(x, z)$, when it exists, is unique $P_{z \mid x}$-a.s. for all $x$.

We shall work in stages, recalling first existing identification results in case of scalar $z$ and clarifying which features we intend to extend and how. The guiding principle 
will be the characterization of shape restrictions on the function $V$ that emulate single crossing and monotonicity restrictions in the scalar case and remain just identifying in the multi-attribute case.

\section{Single market identification With SCALAR ATtRibute}

We first recall and reformulate results of Heckman, Matzkin, and Nesheim (2010) on identification of single attribute hedonic models. Suppose, for the purpose of this section, that $d_{\varepsilon}=d_{z}=1$, so that unobserved heterogeneity is scalar, as is the quality dimension. Suppose further (for ease of exposition) that $\zeta$ is twice continuously differentiable in $z$ and $\varepsilon$ and that $V$ is twice continuously differentiable in $z$. Consumers take price schedule $p(z)$ as given and choose quality $z$ to maximize $\zeta(x, \varepsilon, z)-V(x, z)$. We impose a single crossing condition on $\zeta$.

Assumption 3 (Spence-Mirlees). For all $x, \varepsilon, z$ of the domain of $P_{\tilde{x}, z}, \zeta_{\varepsilon z}(x, \varepsilon, z)>0$.

The first order condition of the consumer problem yields

$$
\zeta_{z}(x, \varepsilon, z)=V_{z}(x, z)
$$

which, under Assumption 3, implicitly defines an inverse demand function $z \mapsto \varepsilon(x, z)$, which specifies which unobserved type consumes quality $z$. Combining the second order condition $\zeta_{z z}(x, \varepsilon, z)<V_{z z}(x, z)$ and further differentiation of (1), i.e., $\zeta_{z z}(x, \varepsilon, z)+\zeta_{\varepsilon z}(x, \varepsilon, z) \varepsilon_{z}(x, z)=V_{z z}(x, z)$, yields

$$
\varepsilon_{z}(x, z)=-\frac{-V_{z z}(x, z)+\zeta_{z z}(x, \varepsilon, z)}{\zeta_{\varepsilon z}(x, \varepsilon, z)}>0 .
$$

Hence the inverse demand is increasing and is therefore identified as the unique increasing function that maps the distribution $P_{z \mid x}$ to the distribution $P_{\varepsilon}$, namely the quantile transform. Denoting $F$ the cumulative distribution function corresponding to the distribution $P$, we therefore have identification of inverse demand according to 
the strategy put forward in Matzkin (2003) as:

$$
\varepsilon(x, z)=F_{\varepsilon}^{-1}\left(F_{z \mid x}(z \mid x)\right) .
$$

The single crossing condition of Assumption 3 on the consumer surplus function $\zeta(x, \varepsilon, z)$ yields positive assortative matching, as in the Becker (1973) classical model. Consumers with higher taste for quality $\varepsilon$ will choose higher qualities in equilibrium and positive assortative matching drives identification of demand for quality. The important feature of Assumption 3 is injectivity of $\zeta_{z}(x, \varepsilon, z)$ relative to $\varepsilon$ and a similar argument would have carried through under $\zeta_{z \varepsilon}(x, \varepsilon, z)<0$, yielding negative assortative matching instead.

Once inverse demand is identified, the function $V(x, z)$, hence the utility function $\bar{U}(x, z)=p(z)-V(x, z)$, can be recovered up to a constant by integration of the first order condition (1):

$$
V(x, z)=\int_{0}^{z} \zeta_{z}\left(x, \varepsilon\left(x, z^{\prime}\right), z^{\prime}\right) d z^{\prime} .
$$

Unlike the demand function, which is identified without knowledge of the surplus function $\zeta$, as long as the latter satisfies Assumption 3, identification of the preference function $\bar{U}(x, z)$ does require a priori knowledge of the function $\zeta$. This includes existing results in this literature. Ekeland, Heckman, and Nesheim (2004) cover the special case of linear marginal utility, where $\zeta(x, \varepsilon, z)=z \varepsilon$. In that case, $\varepsilon(x, z)$ increasing in z, maximizes, by the classical Hardy, Littlewood, and Pólya (1952) inequalities, $\mathbb{E}[z \varepsilon \mid x]$ among all joint distributions for $(z, \varepsilon)$, subject to the marginal restrictions that $z \sim P_{z \mid x}$ and $\varepsilon \sim P_{\varepsilon}$. We thereby recover the efficiency property of positive assortative matching and unlike positive assortative matching, the maximization of $\mathbb{E}[z \varepsilon \mid x]$ has a natural extension in the multivariate linear marginal utility case $\zeta(x, \varepsilon, z)=z^{\prime} \varepsilon$, where quality $z$ and taste for quality $\varepsilon$ are conformable vectors. 
We shall examine this case in the next section, before moving to the general extension with possibly nonlinear marginal utility in taste and arbitrary surplus function $\zeta(x, \varepsilon, z)$.

\section{Single market identification With multiple ATtributes}

4.1. Linear marginal utility. We now turn to the main objective of the paper, which is to derive identifying shape restrictions in the multi attribute case of quality $z \in \mathbb{R}^{d_{z}}$ and unobserved taste $\varepsilon \in \mathbb{R}^{d_{\varepsilon}}$, with $d_{z}=d_{\varepsilon}>1$. We start with linear marginal utility in taste.

Assumption 4 (Linear marginal utility). The surplus function $\zeta(x, \varepsilon, z)=z^{\prime} \varepsilon$.

Under Assumption 4, the consumer maximization problem is that of finding

$$
V^{*}(x, \varepsilon)=\sup _{z}\left\{z^{\prime} \varepsilon-V(x, z)\right\} .
$$

Under suitable regularity, the first order condition yields:

$$
\nabla_{z} V(x, z)=\varepsilon
$$

and the demand function $\varepsilon \mapsto V^{*}(x, \varepsilon)$ is by definition the convex conjugate (also known as Legendre-Fenchel transform) of $V(x, z)$. According to convex duality theory, the conjugate of $V^{*}(x, \varepsilon)$ is $V(x, z)$ itself if and only if $V(x, z)$ is convex. Convexity of $V$ will turn out to be the shape restriction that delivers identification in this linear marginal utility case.

Assumption 5 (Convexity restriction). The function $V(x, z)$ is convex in $z$ for all $x$.

Convexity of $V$ in the univariate case of the previous section is equivalent to monotonicity of demand, or assortative matching, delivered by the single crossing shape restriction on $\zeta$. As discussed in Section 3, positive assortative matching (monotonicity of demand) is difficult to extend to the multi-attribute case, but not the efficiency 
result that comes with positive assortative matching. Imagine a social planner maximizing total surplus over the distribution of heterogeneous customers. The planner's problem is to maximize $\mathbb{E}\left[z^{\prime} \varepsilon \mid x\right]$ over all possible allocations of qualities $z$ to consumer types $\varepsilon$, i.e., over all pairs of random vectors $(z, \varepsilon)$ under the constraint that the marginal distributions $P_{\varepsilon}$ and $P_{z \mid x}$ are fixed. One of the central results of optimal transport theory, Brenier's Theorem (Theorem 3.8 in Villani (2003)), shows precisely that such a planner's problem admits a unique pure allocation as solution, which takes the form of the inverse demand function $\varepsilon=\nabla_{z} V(x, z)$ with $V(x, z)$ convex in $z$. We see thereby that convexity of $V(x, z)$ in $z$ is the shape restriction that delivers identification as summarized in the following theorem, formally proved in the appendix.

Theorem 1 (Identification for linear marginal utility). Under Assumptions 1, 2, 4 and $5, \bar{U}(x, z)$ is nonparametrically identified.

As in the more general case investigated below, $V(x, z)$ can also be shown to be the convex solution to the minimization of $\mathbb{E}[V(x, z) \mid x]+\mathbb{E}\left[V^{*}(x, \varepsilon)\right]$, where $V^{*}$ is the convex conjugate of $V$. This fact provides efficient computation strategies for inference on $\bar{U}(x, z)$, as shown for instance in Aurenhammer, Hoffmann, and Aronov (1998). The identification result of Theorem 3 has implications beyond hedonic equilibrium models, as it provides identification conditions for a general nonlinear nonseparable simultaneous equations econometric model of the form $z=f(x, \varepsilon)$, where the vector of endogenous variables $z$ has the same dimension as the vector of unobserved heterogeneity $\varepsilon$. Theorem 3 shows that in such models, $f$ is nonparametrically identified within the class of gradients of convex functions.

Corollary 1 (Nonlinear simultaneous equations). The simultaneous equations model $z=f(x, \varepsilon)$, with $z, \varepsilon \in \mathbb{R}^{d_{z}}$ and $x \in \mathbb{R}^{d_{x}}$, is nonparametrically identified under the following conditions.

(1) $f$ is the gradient of a convex function. 
(2) The errors $\varepsilon$ are independent of regressors: $\varepsilon \Perp x$.

(3) $P_{\varepsilon}$ is known and $P_{z \mid x}$ is absolutely continuous with respect to Lebesgue measure.

In the univariate case, gradients of convex functions are the increasing functions, so that our identifying shape restriction directly generalizes monotonicity in Matzkin (2003).

4.2. Nonlinear marginal utility. The identification result of Theorem 3 can be easily extended to allow for variation in the quality-unobserved taste interaction with observed type $x$ as in $\zeta(x, \varepsilon, z)=\phi(z)^{\prime} \psi(x, \varepsilon)$, where $\phi$ and $\psi$ are known functions and $\phi$ is invertible. Going beyond this requires mild regularity conditions on the function $\zeta$ specifying the quality-unobserved taste interaction as a function of $x$.

Assumption 6. For all $x, \zeta(x, \varepsilon, z)$ satisfies the following: $(i) \zeta(x, \varepsilon, z)$ is continuous as a function of $(\varepsilon, z)$ and bounded above; (ii) $\zeta(x, \varepsilon, z)$ is everywhere subdifferentiable as a function of $z$ for all $\varepsilon$; and (iii) $\zeta(x, \varepsilon, z)$ is locally Lipschitz as a function of $z$ uniformly in $\varepsilon$.

In the appendix, definitions are recalled (Definitions 6 and 7) and the assumption discussed, including some simple sufficient conditions. Recalling our notation $V(x, z)=p(z)-\bar{U}(x, z)$, the consumer's program is to choose quality vector $z$ to maximize

$$
\sup _{z}\{\zeta(x, \varepsilon, z)-V(x, z)\}
$$

In the one dimensional case, single crossing condition $\zeta_{\varepsilon z}(x, \varepsilon, z)>0$ delivered identification of inverse demand. We noted that the sign of the single crossing condition was not important for the identification result, rather the following implication of single crossing was.

Assumption 7 (Twist condition). For all $x$ and $z$ where it is defined, the gradient $\nabla_{z} \zeta(x, \varepsilon, z)$ of $\zeta(x, \varepsilon, z)$ in $z$ is injective as a function of $\varepsilon$. 
Assumption 7, unlike the single crossing condition, is well defined in the multivariate case, and we shall show, using recent developments in optimal transport theory, that it continues to deliver the desired identification in the multivariate case. Before stating the theorem, we provide more intuition by further developing the parallel between this general case and the univariate and multivariate linear marginal utility cases covered so far.

Consider, as before, the hedonic market from the point of view of a social planner, who allocates qualities $z$ to tastes $\varepsilon$ in a way that maximizes total consumer surplus. The distribution of consumer tastes is $P_{\varepsilon}$ and the distribution of qualities traded at equilibrium is $P_{z \mid x}$. For fixed observable type $x$, the variable surplus of a match between unobserved taste $\varepsilon$ and quality $z$ is $\zeta(x, \varepsilon, z)$. Hence, the planner's problem is find an allocation of qualities to tastes, in the form of a joint probability $\mu$ over the pair of random vectors $(\varepsilon, z)$, so as to maximize $\mathbb{E}_{\mu}[\zeta(x, \varepsilon, z) \mid x]$ under the constraint that $\varepsilon$ has marginal distribution $P_{\varepsilon}$ and that $z$ has marginal distribution $P_{z \mid x}$. This planner's problem

$$
\max _{\mu} \mathbb{E}_{\mu}[\zeta(x, \varepsilon, z) \mid x] \text { subject to } \varepsilon \sim P_{\varepsilon}, z \sim P_{z \mid x}
$$

is equal to its dual

$$
\min _{V, W} \mathbb{E}[W(x, \varepsilon) \mid x]+\mathbb{E}[V(x, z) \mid x] \text { subject to } W(x, \varepsilon)+V(x, z) \geq \zeta(x, \varepsilon, z)
$$

and both primal (4) and dual (5) are attained under the conditions of the MongeKantorovitch Theorem (Theorem 1.3 of Villani (2003), see also Rachev and Rüschendorf (1998) for an account). Notice that the constraint in (5) can be written as

$$
W(x, \varepsilon)=V^{\zeta}:=\sup _{z}\{\zeta(x, \varepsilon, z)-V(x, z)\}
$$

so that $W(x, \varepsilon)$ is a candidate for the demand function mapping tastes $\varepsilon$ into qualities $z$ derived from the consumer's program (3). (6) defines a generalized notion of convex conjugation, discussed in the Appendix, which can be inverted, similarly to convex 
conjugation, into:

$$
\left(V(x, z)^{\zeta}\right)^{\zeta}=\sup _{\varepsilon}\left\{\zeta(x, \varepsilon, z)-V^{\zeta}(x, \varepsilon)\right\}
$$

Definition 2 ( $\zeta$-convexity). A function $V$ is called $\zeta$-convex if and only if $\left(V^{\zeta}\right)^{\zeta}=V$.

$\zeta$-convexity, therefore, is a shape restriction that directly generalizes the convexity restriction of Assumption 5 of the linear marginal utility case.

Assumption 8 ( $\zeta$-convexity). $V(x, z)$ is $\zeta$-convex as a function of $z$ for all $x$.

Under Assumptions 1, 6, 7 and 8, we show with optimal transport theory (mainly Theorem 10.28 of Villani (2009)), that there exists a unique allocation of qualities to tastes $z \mapsto \varepsilon(x, z)$ that maximes the consumer problem (7). Moreover, this allocation is such that markets clear, since $\varepsilon(x, z)$ is distributed according to $P_{\varepsilon}$ when $z$ is distributed according to $P_{z \mid x}$. The following heuristic application of the envelope theorem explains why this is so. By the envelope theorem applied to $V(x, z)=\sup _{\varepsilon}\left\{\zeta(x, \varepsilon, z)-V^{\zeta}(x, \varepsilon)\right.$, for a small variation $\delta V^{\zeta}$ of $V^{\zeta}$, the variation in $V$ is $\delta V(x, z)=-\delta V^{\zeta}(x, \varepsilon(x, z), z)$. Plugging the latter into the first order condition for (5) yields $\mathbb{E}\left[\delta V^{\zeta}(x, \varepsilon)\right]=\mathbb{E}\left[\delta V^{\zeta}(x, \varepsilon(x, z))\right]$. The latter holds for any small variation $\delta V^{\zeta}$, so that the distribution of allocation $\varepsilon(x, z)$ is the same as the exogenously given distribution of unobserved tastes $P_{\varepsilon}$, so that the market clears.

Finally, once the allocation (inverse demand function) $\varepsilon(x, z)$ is identified, $V(x, z)$ satisfies the first order condition

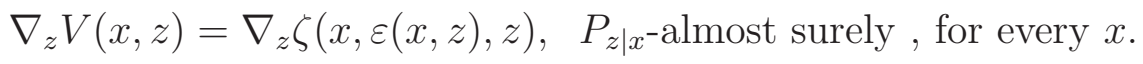

The latter determines $V(x, z)$, and therefore $\bar{U}(x, z)$, up to a constant. We are now ready to state our main theorem, relating the Twist condition and the $\zeta$-convex shape restriction to nonparametric identification of preferences.

Theorem 2 (Identification of preferences). Under Assumptions 1, 2, 6, 7 and 8, $\bar{U}(x, z)$ is nonparametrically identified. 
As before, efficient computation of $\bar{U}(x, z)=p(z)-V(x, z)$ is based on the identification of $V$ as the solution to the optimization problem $\inf _{V}\left[\mathbb{E} V(x, z)+\mathbb{E} V^{\zeta}(x, \varepsilon)\right]$, where $V^{\zeta}$ is defined in (6). Again, the identification result of Theorem 2 has ramification beyond the framework of hedonic equilibrium models. Indeed, it provides the just identifying shape restriction for general consumer problems with multivariate unobserved preference heterogeneity, where consumers choose within a universe of goods, differentiated along more than one dimension. Theorem 2 tells us that the shape of interactions between good qualities and unobserved tastes governs the shape restriction that just identifies the utility function.

\section{IDENTIFICATION WITH EXCLUSION RESTRICTIONS}

The identification results in the previous section rely on observations from a single price schedule and the structural functions are just identified under normalization of the distribution of unobserved heterogeneity. Although $\bar{U}(x, z)$ is not identified without such a normalization, or additional restrictions, there are features of preferences that are identified. Consider the linear marginal utility model of Assumption 4.

$$
U(x, \varepsilon, z)=\bar{U}(x, z)+z^{\prime} \varepsilon .
$$

From Theorem 7, the inverse demand $\varepsilon(x, z)=\nabla_{z}[p(z)-\bar{U}(x, z)]$ satisfies the following. For all bounded continuous functions $\zeta$,

$$
\int \zeta(\varepsilon) f_{\varepsilon}(\varepsilon) d \varepsilon=\int \zeta\left(\nabla_{z} p(z, m)-\nabla_{z} \bar{U}(x, z)\right) f_{z \mid x, m}(z \mid x, m) d z .
$$

Hence, taking $\zeta$ equal to the identity in (8) and assuming only that $P_{\varepsilon}$ has mean zero, instead of fixing the whole distribution, yields identification of averaged partial effects $\int \nabla_{z} \bar{U}(x, z) f_{z \mid x, m}(z \mid x, m) d z$ from the fact that $p(z)$ and $f_{z \mid x}$ are identified.

Theorem 3 (Identification of averaged partial effects). Under Assumptions 2, 4, 5 and zero mean unobserved heterogeneity $\varepsilon$, averaged partial effects are identified by 
the following.

$$
\mathbb{E}\left[\nabla_{z} \bar{U}(x, Z) \mid X=x\right]=\mathbb{E}[\nabla p(Z) \mid X=x]
$$

We now go beyond averaged partial effects and show that some features of preferences may now be identified without normalization of the distribution of unobserved heterogeneity, with an exclusion restriction.

We consider hedonic equilibrium under the assumption of additively separable marginal utility.

Assumption 9 (Additively separable marginal utilities). The consumer's preferences are given by $U(x, z, \varepsilon)=\bar{U}(x, z)+z^{\prime} \varepsilon$, with $\bar{U}(x, z):=z^{\prime} \alpha(x)+\beta(z)$ so that $\nabla_{z}[p(z)-$ $\bar{U}(x, z)]=b(z)-\alpha(x)$, with $b(z)=\nabla_{z}[p(z)-\beta(z)]$.

In the scalar case, Ekeland, Heckman, and Nesheim (2004) identify preferences based on the equality:

$$
F_{z \mid x}(z \mid x)=F_{\varepsilon}\left(\nabla_{z}[p(z)-\bar{U}(x, z)]\right)
$$

Differentiation with respect to $x$ and to $z$ respectively allows to eliminate the unknown $F_{\varepsilon}$ and identify $\alpha$ and $\beta$. In the multivariate case, (9) no longer holds and has to be replaced with the Monge-Ampère equation, see page 126 of Villani (2003). We need the following extra regularity assumptions to allow change of variables between $\varepsilon$ and $z$.

Assumption 10 (Regularity). The functions $x \mapsto \alpha(x)$ and $z \mapsto b(z)$ are continuously differentiable, and the density of observed qualities $f_{z \mid x}(z \mid x)$ is differentiable with respect to $x$ and $z$.

Assumption 11 (Strict convexity shape restriction). The function $p(z)-\beta(z)$ is strictly convex. 
Lemma 1 (Monge-Ampère). Under Assumptions 9, 10 and 11 the following holds.

$$
f_{z \mid x}(z \mid x)=f_{\varepsilon}\left(\nabla_{z}[p(z)-\bar{U}(x, z)]\right) \operatorname{det} D^{2}[p(z)-\bar{U}(x, z)] .
$$

In the Monge-Ampère equation, additive separability in individual and good characteristics in consumer preferences ensures that individual characteristics do not appear in the Jacobian for the change of variables from $\varepsilon$ to $z$. Hence, differencing or differentiating the Monge-Ampère equation with respect to $x$ eliminates the Jacobian term, so that further differentiation eliminates the unknown unobserved taste distribution $f_{\varepsilon}$.

The Monge-Ampère equation yields:

$$
\begin{aligned}
f_{z \mid x}(z \mid x) & =f_{\varepsilon}\left(\nabla_{z}[p(z)-\bar{U}(x, z)]\right) \operatorname{det} D^{2}[p(z)-\bar{U}(x, z)] \\
& =f_{\varepsilon}(b(z)-\alpha(x)) \operatorname{det} J_{b}(z)
\end{aligned}
$$

From the latter, we have identification of:

$$
\frac{f_{\varepsilon}\left(b(z)-\alpha\left(x_{1}\right)\right)}{f_{\varepsilon}\left(b(z)-\alpha\left(x_{2}\right)\right)}=\frac{f_{z \mid x}\left(z \mid x_{1}\right)}{f_{z \mid x}\left(z \mid x_{2}\right)}
$$

for each $x_{1}, x_{2}$ such that the denominators are positive, and by differentiation with respect to $x$, we have identification of:

$$
J_{\alpha}(x) \frac{\nabla f_{\varepsilon}(b(z)-\alpha(x))}{f_{\varepsilon}(b(z)-\alpha(x))}=\nabla_{x} \ln f_{z \mid x}(z \mid x) .
$$

Finally, differentiating (10) with respect to $z$ yields identification of the following.

$$
\begin{aligned}
J_{b}(z)\left[\frac{\nabla f_{\varepsilon}\left(b(z)-\alpha\left(x_{1}\right)\right)}{f_{\varepsilon}\left(b(z)-\alpha\left(x_{1}\right)\right)}\right. & \left.-\frac{\nabla f_{\varepsilon}\left(b(z)-\alpha\left(x_{2}\right)\right)}{f_{\varepsilon}\left(b(z)-\alpha\left(x_{2}\right)\right)}\right] \\
= & \nabla_{z}\left[\ln f_{z \mid x}\left(z \mid x_{1}\right)-\ln f_{z \mid x}\left(z \mid x_{2}\right)\right] .
\end{aligned}
$$

(11) and (12) together yield identification of the following, when properly defined.

$$
\begin{aligned}
J_{b}(z)\left[J_{\alpha}\left(x_{1}\right)^{-1} \nabla_{x} \ln f_{z \mid x}\left(z \mid x_{1}\right)\right. & \left.-J_{\alpha}\left(x_{2}\right)^{-1} \nabla_{x} \ln f_{z \mid x}\left(z \mid x_{2}\right)\right] \\
= & \nabla_{z}\left[\ln f_{z \mid x}\left(z \mid x_{1}\right)-\ln f_{z \mid x}\left(z \mid x_{2}\right)\right] .
\end{aligned}
$$


Identification of $J_{b}$ requires inverting (13), hence a final assumption.

Assumption 12. There exists a collection of values of consumer characteristics $\left(x_{1}^{1}, \ldots, x_{1}^{d_{z}}, x_{1}^{1}, \ldots, x_{1}^{d_{z}}\right)$, not necessarily all distinct, such that the $d_{z} \times d_{z}$ matrix $A_{\alpha}$ with column vectors:

$$
J_{\alpha}\left(x_{1}^{j}\right)^{-1} \nabla_{x} \ln f_{z \mid x}\left(z \mid x_{1}^{j}\right)-J_{\alpha}\left(x_{2}^{j}\right)^{-1} \nabla_{x} \ln f_{z \mid x}\left(z \mid x_{2}^{j}\right) \text {, with } j=1, \ldots, d_{z} \text {, }
$$

is well defined and invertible.

Under Assumption 12, denoting $\mathbf{h}(z)$ the vector with coordinates $\nabla_{z}\left[\ln f_{z \mid x}\left(z \mid x_{1}\right)-\right.$ $\left.\ln f_{z \mid x}\left(z \mid x_{2}\right)\right], J_{b}$ is identified as $J_{b}(z)=A_{\alpha}^{-1} \mathbf{h}(z)$. Since prices of good qualities are observed, identification of $J_{b}(z)$ is equivalent to identification of the Hessian matrix of $\beta$, so that we can summarize the discussion above with the following theorem.

Theorem 4. Under Assumptions 9, 10, 11 and 12, the cross partial effects

$$
\frac{\partial^{2} \beta(z)}{\partial z_{i} \partial z_{j}}, \quad i, j=1, \ldots, d_{z},
$$

are identified, when the effect $\alpha(x)$ of consumer characteristics $\left(x_{i}^{1}, \ldots, x_{i}^{d_{z}}\right)$, for $i=$ 1,2 , on preferences is known.

(13) can also be viewed as a partial identification result, as it delivers partial identification of the pair $\left(J_{\alpha}, J_{b}\right)$, from which Theorem ?? is obtained as a cut of the identified set at the known $J_{\alpha}$.

\section{Appendix}

Throughout the appendix, when there is no ambiguity, we drop the conditioning variable $x$ from the notation and consider the theory of optimal transportation of distribution $P_{z}$ of quality vector $z \in \mathbb{R}^{d}$ to distribution $P_{\varepsilon}$ of vector of unobserved tastes $\varepsilon \in \mathbb{R}^{d}$. 
Kantorovich problem. We first consider the Kantorovich problem, which is the probabilistic allocation of qualities to tastes so as to maximize total surplus, where the surplus of a pair $(\varepsilon, z)$ is given by the function $\zeta(\varepsilon, z)$, and the marginal distributions of qualities $P_{z}$ and tastes $P_{\varepsilon}$ are fixed constraints. We therefore define the set of allocation that satisfy the constraints.

Definition 3 (Probabilities with given marginals). We denote $\mathcal{M}\left(P_{\varepsilon}, P_{z}\right)$ the set of probability measures on $\mathbb{R}^{d} \times \mathbb{R}^{d}$ with marginal distributions $P_{\varepsilon}$ and $P_{z}$.

With this definition, we can formally state the Kantorovitch problem as follows.

$$
(\mathrm{PK})=\sup _{\pi \in \mathcal{M}\left(P_{\varepsilon}, P_{z}\right)} \int \zeta(\varepsilon, z) d \pi(\varepsilon, z)
$$

If we consider the special case of surpluses that are separable in $\varepsilon$ and $z$ and dominate $\zeta(\varepsilon, z)$, i.e., of the form $W(\varepsilon)+V(z) \geq \zeta(\varepsilon, z)$, the integral yields $\int W(\varepsilon) d P_{\varepsilon}(\varepsilon)+\int V(z) d P_{z}(z)$. We denote $\Phi_{\zeta}$ the set of such functions.

Definition 4 (Admissible set). A pair of function $(W, V)$ on $\mathbb{R}^{d}$ belongs to the admissible set $\Phi_{\zeta}$ if and only if $W \in L^{1}\left(P_{\varepsilon}\right), V \in L^{1}\left(P_{z}\right)$ and $W(\varepsilon)+V(z) \geq \zeta(\varepsilon, z)$ for $P_{\varepsilon}$ almost all $\varepsilon$ and $P_{z}$ almost all z.

The integral over separable surpluses

$$
(\mathrm{DK})=\inf _{(W, V) \in \Phi_{\zeta}} \int W(\varepsilon) d P_{\varepsilon}(\varepsilon)+\int V(z) d P_{z}(z)
$$

will in general yield a weakly larger total surplus than (PK), but it turns out that under very weak conditions, the two coincide.

Theorem 5 (Kantorovich duality). If $\zeta$ is upper semi-continuous, then $(P K)=(M K)$ and there exists an allocation $\pi \in \mathcal{M}\left(P_{\varepsilon}, P_{z}\right)$ that achieves the maximum in (PK).

A proof of the Kantorovich duality theorem can be found in Chapter 1 of Villani (2003). We give here the intuition of the result based on the exchange of infimum and supremum operations. First, we notice that under the conditions of Assumption 6 in the main text, $\zeta$ is a continuous function, so that the mapping $\mu \mapsto \int \zeta d \mu$ is continuous in the weak-* topology. Since $\mathcal{M}\left(P_{\varepsilon}, P_{z}\right)$ is weak-* compact, the maximum in (PK) is achieved for some $\pi$ by the Weierstrass Theorem. Hence, an optimal allocation exists. however, continuity of $\zeta$ is not necessary. 
To see the duality result, denote $\chi_{A}(x)=0$ if $x \in A$ and $-\infty$ otherwise. Then, we verify that

$$
\chi_{\mathbb{M}\left(P_{\varepsilon}, P_{z}\right)}=\inf _{(W, V)}\left\{\int W(z) d P_{\varepsilon}(\varepsilon)+\int V(z) d P_{z}(z)-\int(W(\varepsilon)+V(z)) d \pi(\varepsilon, z)\right\},
$$

where the infimum is over all integrable functions, say. Now we can rewrite (PK) as follows:

$$
(\mathrm{PK})=\inf _{\pi}\left\{\int \zeta(\varepsilon, z) d \pi(\varepsilon, z)+\chi_{\mathcal{M}\left(P_{\varepsilon}, P_{z}\right)}(\pi)\right\},
$$

where the supremum is taken over all joint probability measures. Assuming the infimum and supremum operations can be switched yields:

$$
\begin{aligned}
&(\mathrm{PK})= \inf _{(W, V)} \sup _{\pi}\left\{\int \zeta(\varepsilon, z) d \pi(\varepsilon, z)\right. \\
&\left.\quad+\int W(z) d P_{\varepsilon}(\varepsilon)+\int V(z) d P_{z}(z)-\int(W(\varepsilon)+V(z)) d \pi(\varepsilon, z)\right\} \\
&=\inf _{(W, V)}\left\{\int W(z) d P_{\varepsilon}(\varepsilon)+\int V(z) d P_{z}(z)\right. \\
&\left.\quad-\inf _{\pi} \int(W(\varepsilon)+V(z)-\zeta(\varepsilon, z)) d \pi(\varepsilon, z)\right\} .
\end{aligned}
$$

Consider the second infimum in the last display. If the function $W(\varepsilon)+V(z)-\zeta(\varepsilon, z)$ takes a negative value, then, choosing for $\pi$ the Dirac mass at that point will yield an infimum of $-\infty$. Therefore, we have:

$$
\inf _{\pi} \int(W(\varepsilon)+V(z)-\zeta(\varepsilon, z)) d \pi(\varepsilon, z)=\chi_{\Phi_{\zeta}}(W, V)
$$

so that

$$
(\mathrm{PK})=\inf _{(W, V)}\left\{\int W(z) d P_{\varepsilon}(\varepsilon)+\int V(z) d P_{z}(z)-\chi_{\Phi_{\zeta}}(W, V)\right\}=(\mathrm{DK})
$$

as required.

$\zeta$-convexity theory. In the dual formulation (DK) of the Kantorovich problem, the constraint $(W, V) \in$ $\Phi_{\zeta}$ can be rewritten

$$
W(\varepsilon)=\sup _{z}(\zeta(\varepsilon, z)-V(z)) .
$$

In the special case, where $\zeta(\varepsilon, z)=\varepsilon^{\prime} z, W(\varepsilon):=\sup _{z}\left(\varepsilon^{\prime} z-V(z)\right)$ defines the Fenchel-Legendre transform of $V$. $W$ is a convex function, and as such, it is also known as convex conjugate of $V$, denoted $V^{*} . V$ is convex if and only if $V(z)=V^{* *}(z):=\sup _{\varepsilon}\left(\varepsilon^{\prime} z-V^{*}(\varepsilon)\right)$.

Similarly, a generalized notion of convex duality can be defined from the Kantorovich problem. A function is called $\zeta$-convex if and only if it can be written in the form (14). 
Definition 5 ( $\zeta$-convexity). A function $W$ is called $\zeta$-convex if and only if there exists $V$ such that (14) holds. The $\zeta$-transform of $W$ is the function $W^{\zeta}$ defined by:

$$
W^{\zeta}(z)=\sup _{\varepsilon}(\zeta(\varepsilon, z)-W(\varepsilon)) .
$$

We can state the definition of $\zeta$-convexity given in the main text as a straightforward characterization.

Lemma 2. A function $V$ is $\zeta$-convex if and only if $V^{\zeta \zeta}=V$.

Proof of Lemma 2. By definition, we have

$$
W^{\zeta \zeta \zeta}(\varepsilon)=\sup _{z} \inf _{\tilde{\varepsilon}} \sup _{\tilde{z}}(\zeta(\varepsilon, z)-\zeta(\tilde{\varepsilon}, z)+\zeta(\tilde{\varepsilon}, \tilde{z})-W(\tilde{z})) .
$$

Choosing $\tilde{\varepsilon}=\varepsilon$ yields $W^{\zeta \zeta \zeta}(\varepsilon) \leq W^{\zeta}(\varepsilon)$, while choosing $\tilde{z}=z$ yields $W^{\zeta \zeta \zeta}(\varepsilon) \geq W^{\zeta}(\varepsilon)$. Hence $W^{\zeta \zeta \zeta}(\varepsilon)=W^{\zeta}(\varepsilon)$. If $W$ is $\zeta$-convex, then there is a $V$ such that $W=V^{\zeta}$, hence $W^{\zeta \zeta}=V^{\zeta \zeta \zeta}=$ $V^{\zeta}=W$. Conversely, if $W=W^{\zeta \zeta}$, then $W$ is $\zeta$-convex as the $\zeta$-transform of $W^{\zeta}$.

Returning to the Kantorovich duality result, we now see that the dual is also achieved.

Theorem 6 (Kantorovich duality (continued)). If $(P K)<\infty$ and there exist integrable functions $\zeta^{\varepsilon}$ and $\zeta^{z}$ such that $\zeta(\varepsilon, z) \geq \zeta^{\varepsilon}(\varepsilon)+\zeta^{z}(z)$, then there exists a $\zeta$-convex function $V$ such that

$$
\int V^{\zeta}(\varepsilon) d P_{\varepsilon}(\varepsilon)+\int V(z) d P_{z}(z)
$$

achieves (DK). In addition, if $\pi$ is an optimal allocation, i.e., achieves $(P K)$, and $\left(V^{\zeta}, V\right)$ is an optimal $\zeta$-conjugate pair, i.e., achieves (DK), then

$$
V^{\zeta}(\varepsilon)+V(z)=\zeta(\varepsilon, z), \quad \pi \text {-a.s. }
$$

Idea of the proof. The proof can be found in Chapter 5 of Villani (2009). The last statement of Theorem 5 is easy to see. If $\pi$ achieves $(\mathrm{PK})$ and $\left(V^{\zeta}, V\right)$ achieves $(\mathrm{DK})$, then, as $(\mathrm{PK})=(\mathrm{DK})$ by Theorem 4, we have $\int\left[V^{\zeta}(\varepsilon)+V(z)-\zeta(\varepsilon, z)\right] d \pi(\varepsilon, z)=0$. The integrand is non negative, since $\left(V^{\zeta}, V\right) \in \Phi_{\zeta}$. Hence, $V^{\zeta}+V=\zeta, \pi$-almost surely, as desired. The proof of existence of an optimal pair of $\zeta$-convex functions achieving (DK) revolves around the notion of cyclical monotonicity.

In view of the above, if $(\phi, \psi)$ achieve (DK) and a sequence of pairs $\left(\varepsilon_{i}, z_{i}\right)_{i=1, \ldots, m}$ belong to the support of the optimal allocation $\pi$, then $\phi\left(\varepsilon_{i}\right)+\psi\left(z_{i}\right)=\zeta\left(\varepsilon_{i}, z_{i}\right)$ for each $i=1, \ldots, m$. On the other hand, since $(\phi, \psi) \in \Phi_{\zeta}$, we have $\phi\left(\varepsilon_{i}\right)+\psi\left(z_{i+1}\right) \geq \zeta\left(\varepsilon_{i}, z_{i+1}\right)$ for each $i=1, \ldots, n-1$, and $\phi\left(\varepsilon_{m}\right)+\psi(z) \geq \zeta\left(\varepsilon_{m}, z\right)$ for an arbitrary $z$. Substracting and adding up yields $\psi(z) \geq \psi\left(z_{1}\right)+$ 
$\left[\zeta\left(\varepsilon_{m}, z\right)-\zeta\left(\varepsilon_{m}, z_{m}\right)\right]+\ldots+\left[\zeta\left(\varepsilon_{1}, z_{2}\right)-\zeta\left(\varepsilon_{1}, z_{1}\right)\right]$. Since the functions in the pair $(\phi, \psi)$ are only determined up to a constant, normalize $\psi\left(z_{1}\right)=0$ and define $V$ as the supremum of all functions $\psi$ satisfying $\psi(z) \geq\left[\zeta\left(\varepsilon_{m}, z\right)-\zeta\left(\varepsilon_{m}, z_{m}\right)\right]+\ldots+\left[\zeta\left(\varepsilon_{1}, z_{2}\right)-\zeta\left(\varepsilon_{1}, z_{1}\right)\right]$ over all choices of $\left(\varepsilon_{i}, z_{i}\right)_{i=1, \ldots, m}$ in the support of $\pi$ and all $m \geq 0$. It turns out that $V^{\zeta}(\varepsilon)+V(z)=\zeta(\varepsilon, z), \pi$-almost surely, so that integration over $\pi$ yields the fact that $\left(V^{\zeta}, V\right)$ achieves (DK) as desired.

Let us now look more closely at the regularity properties of $\zeta$-convex functions, as they drive our identification results. First recall definitions of the regularity conditions of Assumption 6.

Definition 6 (Lipschitz continuity). Let $U$ be an open subset of $\mathbb{R}^{d}$ and $f$ a function on $U$. (i) $f$ is said to be Lipschitz if there exists $L<\infty$ such that for all $x, z \in U,|f(z)-f(x)| \leq L\|z-x\|$. (ii) $f$ is said to be locally Lipschitz if, for any $x_{0} \in U$, there is a neighborhood of $x_{0}$ on which $f$ is Lipschitz.

Locally Lipschitz functions on $\mathbb{R}^{d}$ are differentiable almost everywhere according to a celebrated result known as Rademacher's Theorem. A proof can be found in Villani (2009) Theorem 10.8(ii).

Theorem 7 (Rademacher). Let $U$ be an open subset of $\mathbb{R}^{d}$ and $f$ a function on $U$. If $f$ is locally Lipschitz, then it is differentiable almost everywhere.

Definition 7 (Subdifferentiability). Let $U$ be an open subset of $\mathbb{R}^{d}$ and $f$ a function on $U$. $f$ is said to be subdifferentiable at $x \in U$ with subgradient $p$ if

$$
f(z) \geq f(x)+p^{\prime}(z-x)+o(\|z-x\|) .
$$

The (convex) set of subgradients at $x$ is called subdifferential of $f$ at $x$ and denoted $\partial^{-} f(x)$. Superdifferentiability, supergradients and superdifferentials are defined symetrically.

Proposition 1 (Differentiability). Let $U$ be an open subset of $\mathbb{R}^{d}$ and $f$ a function on $U$. If $f$ is sub- and superdifferentiable at $x \in U$, or equivalently, if $f$ has a subgradient and a supergradient at $x$, then $f$ is differentiable at $x$, or equivalently, the subgradient and supergradient are unique and equal to the gradient denoted $\nabla f(x)$.

Proof of Proposition 2. Let $p$ (resp. $q$ ) be a sub- (resp. super-) gradient of $f$ at $x$. By definition, $f(z)-f(x) \geq p^{\prime}(z-x)+o(\|z-x\|)$ and $f(z)-f(x) \leq q^{\prime}(z-x)+o(\|z-x\|)$, so that $(p-q)^{\prime}(z-x) \leq$ $o(\|z-x\|)$. Hence:

$$
\lim _{z \rightarrow x ; z \neq x}(p-q)^{\prime} \frac{z-x}{\|z-x\|}=0 .
$$


Since the vector $(z-x) /\|z-x\|$ can take arbitrary values on the unit sphere as $z \rightarrow x$, it follows that $p=q$ as desired.

We now turn to a specific regularity properties of $\zeta$-convex functions, which are ingredients in the proof of Theorem 2 .

Lemma 3 (Differentiability of $\zeta$-convex functions). Suppose $\zeta$ satisfies Assumptions 6 and 7 , then any $\zeta$-convex function is almost everywhere differentiable.

Proof of Lemma 3. Since $\zeta$ is locally Lipschitz uniformly in $\varepsilon$, for any neighborhood $U$ of any $z_{0}$, there is $L<\infty$ such that, for all $\varepsilon$, all $z \in U,\left|\zeta(\varepsilon, z)-\zeta\left(\varepsilon, z_{0}\right)\right| \leq L\left\|z-z_{0}\right\|$. If $V$ is a $\zeta$-convex function, then $V(z)=\sup _{\varepsilon}\left\{\zeta(\varepsilon, z)-V^{\zeta}(\varepsilon)\right\}$ and $\left|V(z)-V\left(z_{0}\right)\right| \leq L\left\|z-z_{0}\right\|$, so $V$ is locally Lipschitz. By Theorem $6, V$ is differentiable almost everywhere.

The quadratic case and Brenier's Theorem. In the special case of Assumption 4, where $\zeta(\varepsilon, z)=z^{\prime} \varepsilon$, the planner's program $(\mathrm{PK})$ writes

$$
\sup _{\pi \in \mathcal{M}\left(P_{\varepsilon}, P_{z}\right)} \int z^{\prime} \varepsilon d \pi(\varepsilon, z)
$$

and the set $\Phi_{\zeta}$ becomes

$$
\Phi=\left\{(W, V): W(\varepsilon)+V(z) \geq z^{\prime} \varepsilon\right\} .
$$

The pair $\left(V^{*}, V\right) \in \Phi$ defined by

$$
\begin{aligned}
V^{*}(\varepsilon) & =\sup _{z}\left\{z^{\prime} \varepsilon-V(z)\right\}, \\
V(z) & =\sup _{\varepsilon}\left\{z^{\prime} \varepsilon-V^{*}(\varepsilon)\right\}
\end{aligned}
$$

achieves the minimum in the dual problem (DK). Notice that $V$ and $V^{*}$ are standard FenchelLegendre convex conjugates of each other and that $V=V^{* *}$ and is hence convex.

In this case, $\nabla V(z)=\nabla \zeta_{z}(\varepsilon, z)$ simplifies to $\nabla V(z)=\varepsilon$, which guarantees uniqueness and purity of the optimal assignment $\varepsilon=\nabla V(z)$, where $V$ is convex. As a corollary, $\nabla V$ is a $P_{z}$-almost surely uniquely determined gradient of a convex function.

Theorem 8 (Brenier). Suppose $P_{z}$ is absolutely continuous with respect to Lebesgue measure and that $P_{\varepsilon}$ and $P_{z}$ have finite second order moments. Then, there exists a $P_{z}$-almost surely unique map of the form $\nabla V$, where $V$ is convex, such that $\int z^{\prime} \nabla V(z) d P_{z}(z)$ achieves the maximum in (PK) with $\zeta(\varepsilon, z)=z^{\prime} \varepsilon$. Moreover, $\left(V^{*}, V\right)$ achieves the dual program (DK). 
Note that Theorem 7 is not a direct corollary of Theorem 2 since the boundedness condition of Assumption 6 doesn't hold. The proof can be found in Chapter 2 of Villani (2009).

Proof of Theorem 3. This follows directly from Theorem 7.

Proof of Theorem 2. The proof is adapted from Chapter 10 of Villani (2009). By Theorem 5, there exists a $\zeta$-convex function $V$ such that $\left(V^{\zeta}, V\right)$ solves the dual Kantorovich problem (DK). Let $\pi$ be an allocation that solves the primal Kantorovich problem (PK). We know from Theorem 5 that any $\left(\varepsilon_{0}, z_{0}\right)$ in the support of $\pi$ (quality $z_{0}$ is consumed by type $\varepsilon_{0}$ with non zero probability at the optimal allocation) satisfies $V^{\zeta}\left(\varepsilon_{0}\right)+V\left(z_{0}\right)=\zeta\left(\varepsilon_{0}, z_{0}\right)$. Moreover, we also know that $\left(V^{\zeta}, V\right) \in \Phi_{\zeta}$, so for any other $z, V^{\zeta}\left(\varepsilon_{0}\right)+V(z) \geq \zeta\left(\varepsilon_{0}, z\right)$. Hence $V(z)-V\left(z_{0}\right) \geq \zeta\left(\varepsilon_{0}, z\right)-\zeta\left(\varepsilon_{0}, z_{0}\right)$. Fix $w$ and choose a sequence $z(\eta)=z_{0}+\eta w$ for $\eta \in\left[0, \eta_{0}\right)$. Write $V(z(\eta))-V\left(z_{0}\right) \geq \zeta\left(\varepsilon_{0}, z(\eta)\right)-\zeta\left(\varepsilon_{0}, z_{0}\right)$, divide through by $\eta$ and take the limit. Assume $z_{0}$ is a point of differentiability of $V$. We then obtain the following:

$$
\nabla V\left(z_{0}\right)^{\prime} w=\lim _{\eta \rightarrow 0} \frac{1}{\eta}\left(V(z(\eta))-V\left(z_{0}\right)\right) \geq \lim _{\eta \rightarrow 0} \inf \frac{1}{\eta}\left(\zeta\left(\varepsilon_{0}, z(\eta)\right)-\zeta\left(\varepsilon_{0}, z_{0}\right)\right)
$$

The latter display shows that $\nabla V\left(z_{0}\right)$ is a supergradient of $\zeta\left(\varepsilon_{0}, z\right)$ (as a function of its second $\operatorname{argument}$ ) at $z_{0}$. By Assumption 6, $\zeta$ is subdifferentiable, so it is both sub- and superdifferentiable at $z_{0}$, hence differentiable, by Proposition 2 .

Hence, we shown that $\zeta(\varepsilon, z)$ is differentiable (as a function of its second argument) at $z_{0}$ and that the gradient $\nabla_{z} \zeta\left(\varepsilon, z_{0}\right)$ at $z_{0}$ of $\zeta(\varepsilon, z)$ relative to its second argument is equal to $\nabla V\left(z_{0}\right)$. By Lemma 3 , the latter reasoning is true for almost every $z_{0}$ (hence $P_{z}$-almost every $z_{0}$ under Assumption 2), and all $\varepsilon_{0}$ such that $\left(\varepsilon_{0}, z_{0}\right)$ is in the support of an optimal allocation $\pi$.

By Assumption $7, \nabla_{z} \zeta\left(\cdot, z_{0}\right)$ is injective for each $z_{0}$ where it is defined. Call $\left(\nabla_{z} \zeta\right)^{-1}\left(\cdot, z_{0}\right)$ the inverse of $\nabla_{z} \zeta\left(\cdot, z_{0}\right)$ defined on the range of the latter. By the previous result, we have $\varepsilon_{0}=$ $\left(\nabla_{z} \zeta\right)^{-1}\left(\nabla V\left(z_{0}\right), z_{0}\right)$ for almost all $z_{0}$ and all $\varepsilon_{0}$ such that $\left(\varepsilon_{0}, z_{0}\right)$ is in the support of the optimal assignment $\pi$. Hence, there is a deterministic map $z_{0} \mapsto\left(\nabla_{z} \zeta\right)^{-1}\left(\nabla V\left(z_{0}\right), z_{0}\right)$ relating $\varepsilon_{0}$ to $z_{0}$ on the support of $\pi$, which implies that the joint probability $\pi$ is concentrated on the graph of the latter map. The optimal assignment is pure. Uniqueness comes from the observation that the reasoning above does not depend on the particular choice of optimal assignment $\pi$ at the outset.

Finally, calling $z_{0} \mapsto \varepsilon_{0}\left(z_{0}\right)=\left(\nabla_{z} \zeta\right)^{-1}\left(\nabla V\left(z_{0}\right), z_{0}\right)$ the unique inverse demand mapping identified above, $\nabla V\left(z_{0}\right)$ is a random variable with the same distribution as the uniquely defined random 
variable $\nabla_{z} \zeta\left(\varepsilon_{0}\left(z_{0}\right), z_{0}\right)$. As such, it is uniquely determined $P_{z}$-almost surely, as desired. This completes the proof.

\section{REFERENCES}

Aurenhammer, F., F. Hoffmann, and B. Aronov (1998): "Minkowski-type theorems and least squares clustering," Algorithmica, 20, 61-76.

BAJARI, P., and L. Benkard (2005): "Demand estimation with heterogeneous consumers and unobserved product characteristics: a hedonic approach," Journal of Political Economy, 113, 12391276.

BARTIK, T. (1987): "The estimation of demand parameters in hedonic price models," Journal of Political Economy, 95, 81-88.

Becker, G. (1973): "A Theory of marriage: part I," Journal of Political Economy, 81, 813-846.

Bishop, K., and C. Timmins (2011): "Hedonic prices and implicit markets: Estimating marginal willingness to pay for differentiated products without instrumental variables," NBER Working Paper Series, 17611, 1-38.

Brown, J., and H. Rosen (1982): "On the estimation of structural hedonic price models," Econometrica, 50, 765-769.

Chiappori, P.-A., R. McCann, and L. Nesheim (2010): "Hedonic price equilibria, stable matching, and optimal transport: equivalence, topology, and uniqueness," Economic Theory, 42, 317-354.

Chiong, K., A. Galichon, and M. Shum (2013): "Estimating dynamic discrete choice models via convex analysis," unpublished manuscript.

Choo, E., and A. Siow (2006): "Who marries whom and why?," Journal of Political Economy, $114,175-201$.

EkEland, I. (2010): "Existence, uniqueness and efficiency of equilibrium in hedonic markets with multidimensional types," Economic Theory, 42, 275-315.

Ekeland, I., J. Heckman, and L. Nesheim (2004): "Identification and estimation of hedonic models," Journal of Political Economy, 112, 60-109.

Epple, D. (1987): "Hedonic prices and implicit markets: Estimating demand and supply functions for differentiated products," Journal of Political Economy, 95, 59-80.

Galichon, A., and B. Salanié (2012): "Cupid's invisible hand; social surplus and identification in matching models," unpublished manuscript.

Hardy, G., J. Littlewood, and G. Pólya (1952): Inequalities. Cambridge University Press. 
Heckman, J., R. Matzkin, and L. Nesheim (2010): "Nonparametric identification and estimation of nonadditive hedonic models," Econometrica, 78(5), 1569-1591.

KHAN, S., and K. LANG (1988): "Efficient estimation of structural hedonic systems," International Economic Review, 29, 157-166.

Knothe, H. (1957): "Contributions to the theory of convex bodies," Michigan Mathematical Journal, 4, 39-52.

Matzkin, R. (2003): "Nonparametric estimation of nonadditive random functions," Econometrica, $71,1339-1375$.

MatzKin, R. (2013): "Nonparametric identification in structural econometric models," Annual Review of Economics, 5, 457-486.

Nesheim, L. (2013): "Identification of multidimensional hedonic models," Conference Presentation.

PAss, B. (2011): "Regularity properties of optimal transportation problems arising in hedonic pricing models," unpublished manuscript.

Rachev, S., and L. Rüschendorf (1998): Mass Transportation Problems. Springer, New York.

Rosen, S. (1974): "Hedonic prices and implicit markets: product differentiation in pure competition," Journal of Political Economy, 82, 34-55.

Rosenblatt, M. (1952): "Remarks on a multivariate transformation," The Annals of Mathematical Statistics, 23, 470-472.

Tinbergen, J. (1956): "On the theory of income distribution," Weltwirtschaftliches Archiv, 77, $155-173$.

Villani, C. (2003): Topics in Optimal Transportation. American Mathematical Society, Providence.

Villani, C. (2009): Optimal Transport. Springer, Berlin.

Mit, Sciences-Po, Penn State 